\title{
Traditional Chinese Medicine for Mammary Dysplasia (Fibrocystic Breast Disease): A Case Report
}

\author{
Abeer Elmohandes ${ }^{1}$ \\ ${ }^{1}$ American Academy of Family Physicians, American Medical Association, 11400 Tomahawk Creek Pkwy, \\ Leawood, KS 66211, USA \\ Correspondance: Dr Abeer Elmohandes, American Board Certified Family Medicine /Master Degree of Public \\ Health, Dubai, Dubai healthcare city, PO Box 505005, United Arab Emirates. Tel: 971-4-429-7777, \\ 1-810-837-0812, 971-50-261-7899. FAX: 971-4-429-7788
}

Received: December 8, 2020 Accepted: January 5, 2021 Online Published: January 13, 2021

doi:10.5539/gjhs.v13n2p118

URL: https://doi.org/10.5539/gjhs.v13n2p118

\begin{abstract}
Breast cancer (BC) is one among the most common and fatal malignancies affecting women of all ages, though prognosis are good if caught early. Fibrocystic breast disease, which is interchangeably mentioned as fibrocystic breast, is nothing but a benign (noncancerous) condition which gives a lumpy feeling to women. It is not a rapidly progressing disease or dangerous, but is a constant source of problem to some women.

In this case report, we present a female suffering from mammary dysplasia (MD). She was initially treated with conventional medicine but received no benefit, so shifted to Traditional Chinese medicine (TCM). Here, acupuncture, herbal remedies, and specific instructions for a dietary regimen and bra usage were given. We found that by utilizing this holistic approach to treat the root cause, rather than the symptoms, breast pain was eliminated, cystic lumps and related densities were diminished, and cancer progression was thwarted by pursuing healthy lifestyle modification and paying more attention to diet and exercise. Ultimately, this resulted in a better quality of life and suggests that TCM can be employed successfully to treat MD when conventional medicine has failed.
\end{abstract}

Keywords: acupuncture, breast cancer, fibrocystic disease, herbal remedy, mammary dysplasia, traditional Chinese medicine

\section{Introduction}

Mammary dysplasia (MD) or fibrocystic breast disease is a benign condition that affects breast tissue, rendering it lumpy in texture and giving the impression of tumors inside. It is estimated that $16 \%$ of all female cancers are due to MD (Hornberger et al., 2005), with Mastodynia, Macrocystic disease, and Microcystic disease being the most common types (Copeland, 1963).

Owing to advances in screening technology, survival rates for BC patients have increased dramatically. Mammograms, in particular, are widely used to evaluate breast tumors of benign or malignant origin. Having said this, there are no clear guidelines used to distinguish between the subtypes of benign breast disease except for those classified as proliferative lesions with atypia. The modern (allopathic) medical system has offered a variety theoretical solutions for treating MD (e.g., medical and surgical interventions), while therapies offered by holistic medical systems like Ayurveda, Siddha, Unani, and Traditional Chinese medicine (TCM) have strong traditions and beliefs based more on empirical values. In this case report, we present a woman with MD who was treated successfully with TCM.

\subsection{Aim of the Case Report}

This case report aims to convey the importance of TCM in the management of MD.

\subsection{Research Question}

The case report was presented to solve the following research problem or question. Can TCM be an effective and permanent solution for MD particularly for the pain and quality of life when western medicine doesn't have had a solution?

\section{Methodology}

A 42-year-old female complained of severe breast pain with a 5-year history of insidious onset. She had mild 
tenderness and pain that was intermittent, though often aggravated during menstruation. She explained the discomfort or pain as a feeling of swollen due to pressure from inside and a discomfort in chest wall expansion during silent breathing. To this symptom she had to do deep breathing with a heaving sigh. Also, the patient noted multiple lumps in both breasts and a lump drainage occurring two years prior that led to a diagnosis of fibrocystic breast disease by her gynecologist. Her signs and symptoms were consistent with MD, as her age was between 20 and 50 years and she had a number of painful lumps that changed according to her menstrual cycle. Observational analysis of her breasts discovered no blemishes, contour changes, areas of skin discoloration, or nipple discharge. Upon palpation, she remarked a generalized tenderness in both breasts. The supra-clavicular lymph nodes, the axillary lymph nodes, and the supra-trochlear lymph nodes" were not obvious or non-palpable but presented with tenderness. There were several, discrete nodules or trigger like presentation identified in both breasts, which were thick in consistency and hyper-dense tissue to palpate. They were present in both the upper quadrant and lower quadrant of the breast. Thermography, a non-invasive way to assess breast tissue that spares the patient from trauma, radiation, and high expenses associated with mammograms, revealed only minor changes in surface temperature for both breasts, indicating a low risk of BC. Hence she was prescribed with dietary modification in the form of low caffeine diet. She was advised to wear bras which provided enough support to the breast. The patient prognosis better with these two preliminary modifications, but pain severity remained the same particularly there was no significant improvement during the premenstrual time. Due to such poor prognosis associated with conventional methods, she opted to explore TCM and its emphasis on nutrition for overall health. For about a year, her regular diet comprised of a banana tofu shake and straw berry for breakfast. For lunch she had one vegetable curry and rice or vegetable salad for lunch. Her dinner also contained vegetables with rice and frequently potato. Importantly, her diet did not contain any animal product like dairy products or meat but it was predominantly fruit and vegetable. Alongside her meals, this patient took multi-vitamin/mineral supplements and vitamin $\mathrm{C}$ and $\mathrm{E}$ tablets. Non-steroidal anti-inflammatory drugs were the only allopathic drug she used that too when only she had severe pain

TCM utilizes observation, auscultation, olfaction, interrogation, palpation, and pulse evaluation as the major modes of examination for assessing a condition and predicting prognosis. In using this approach, we obtained helpful information. She reported that both breasts felt distended and painful, and upon palpation based on TCM principles. She reported tenderness along the liver meridian particularly in the distal portion of the leg and also at just below the breast where the liver meridian terminates. Hence, one of the major differences between allopathic medicine and TCM is that the latter considers not only the physical plateau but the mental plateau, giving it a holistic slant. The patient also reported about emotional issues like anger, and memory issues, severe anxiety, along with compromised sleep quality and duration.

Her pulse was wiry, thin, and rapid, while her tongue felt dry and discoursed into red with yellow tinge of coat. She complained of bitter taste in her mouth and said her throat often remained dry, yet she never felt thirsty. She reported that the proportion of urinary output was less compared to the intake and had heavy flow during her menstrual cycle which looked unusually deep red in color.

The allopathic physician advised her to be aware of potential breast injuries as a preventive measure but did never provide her permanent solution to the impairment. Subsequently when the patient was treated with TCM where the following measures were adopted.

\subsection{Diagnosis via Thermography}

Thermography was used to diagnose her MS, as it identifies vascular changes accompanying cancer much earlier than mammography, especially for dense breasts or in young women (American College of Clinical Thermology, 2017). New blood vessel formation is the primary and detectable change that precedes the pathogenesis of cancer. This can be discerned by infrared thermography. Research has shown that almost forty present of female presenting with both symptoms of fibrocystic breasts and positive thermogram have a highest possibility of developing breast cancer within 5 years of time. (Kennedy Lee et al., 2009). On the other hand, subjects with fibrocystic breast with normal values of thermogram very rarely developed $\mathrm{BC}(<3 \%)$. When it comes to mammogram the validity and sensitivity of identifying $\mathrm{BC}$ among the pre-menopausal women $(<50$ years of age) is bleak because of their dense breast tissue which offers a resistance to reading the underlying structures. (Crystal Strano et al., 2003). The failures of mammography in this population have resulted in an unfortunate situation of utilizing biopsies as the valid source to confirm BC. The irony is most of these cases are tested positive for benign type. Also, mammograms are hazardous for the breasts. It is proved that repeated and periodical exposed (since this screening tool relies on radiation) to radiation empirically increase the risk of $\mathrm{BC}$ by at least $1 \%$ for every breast series when completed (Hoekstra, 2001). For instance, a thirty five year old female who undergoes 
mammogram analysis every year could increase her chances of BC by $15 \%$ at age of 50 years. Therefore, a young woman with a positive family history should prefer thermography as a diagnostic tool to identify BC. (Institute of Medicine (US) and National I Council, 2005). For a solution to this diagnostic controversy, the patient was advised to continue with yearly thermograms until age 50, when mammograms would then be added. At this older age, mammography is beneficial for diagnosing $\mathrm{BC}$ at an acute stage of $\mathrm{BC}$ and the patient is at a reduced risk of developing cancer via radiation exposure.

\subsection{Modifications in Diet}

Adhering to a healthy diet is emphasized in this manuscript because $t$ consumption of Dieldrin is directly proportional to the progress and inversely proportional to the prognosis of BC (Snedeker, 2001). Dieldrin is an insecticide often found in non-organic meat products, and high consumption of Dieldrin associates with a heightened risk of cancer. Thus, the patient was advised to avoid eating meat. Moreover, due to her diet being short of fats, she was advised to consume flaxseed oil as a supplement. It is believed that fat is harmful to our health, although we do need it in many areas of the body for immunity, thermoregulation, protection and so on. Fatty acids that are utilized in performing such constructive work are termed essential fatty acids which are present abundantly in nuts, vegetables, fish, and flaxseeds. Their main role is to maintain the integrity of cell walls of neurons, hair cells, skin epithelium and so on. Fats are also involved in the production of prostaglandins, which are nothing but lipids that regulates inflammation. They are derived from animal fats tend to promote inflammation, whereas those from marine fish and healthy oils suppress it

\subsection{Supports for Mental Health in TCM}

The presence of breast masses are always a source of the psychological problem to the female which results in negative emotions that result in the stagnation of energy (Qi) flow in the liver meridian. When this combines with the stagnation of blood, breast masses will develop. Additionally, this scenario results in retarded circulation that causes qi and blood to aggregate. This is the main reason for the feeling of fullness or distension along with pain in $\mathrm{BC}$. This cycled event gets aggravated during the menstrual cycle. On the other hand bitter taste and dryness of tongue, and a feeble pulse signifies that there is a reduction of liver yin. According to the TCM classical theories, $\mathrm{BC}$ is regarded as a pathology that progresses through different stages. The disease always progresses from premenstrual breast pain, advancing to fibrocystic changes, and finally resulting in tumor or BC. Hence, a physician's clear explanation of the condition, proper dietary counseling, and reassurance are perceived as very important in TCM.

\subsection{Administration of Herbal Remedies and Acupuncture}

"Sinensis (Dong quai)", "dioscorea villosa (wild yam"), "caulophyllum thalictroides (blue cohosh)", and "Vitex agnus-castus (chaste-tree berry)" were the herbal remedies of choice for our patient. Acupuncture was also delivered weekly and the liver meridian was treated as an essential source of problem, and the secondary focus was on the area above and below the breasts where the meridian ends which was an effort to restore hepatic function and clear the channels that traverse the breasts.

\subsection{Advice on Bra Usage and Breast Massages}

A tight bra or a supportive bra can result in compromised blood supply to the breast. Hence she was discouraged from wearing one and taught how to massage her breasts for the purpose of increasing lymphatic drainage and diminishing breast pain and cystic lumps. There was significant progress in the pain with the patient reporting a fifty present reduction in overall symptoms she was experiencing since a long time. For the following two months, she was instructed to continue supplementing her diet with flaxseed oil, taking the aforementioned herbal remedies, and receiving weekly acupuncture treatments. In result, her mastalgia dissipated and so she prescribed to undergo acupuncture alternate week for next two months until discontinuing it. Currently, she only takes flaxseed oil and the herbal prescription on account of her good prognosis.

\section{Discussion}

This case report emphasis on monitoring and altering the patient's diet because food has the most profound impact on human health and some foods are well known for invoking immune reactions that pose a threat to longevity. For example, every time a person with an egg allergy eats eggs, their body produces antibodies that attack egg proteins in their bloodstream. The resulting allergic reaction brings about an antigen/antibody complex that needs to be handled by the liver; however, this can cause a burden that if severe enough, will prevent the liver from effectively detoxifying the blood. In this instance, estrogen levels will increase and accelerate fibrocystic changes in breast tissue. Similarly, select foods can also provoke lymphatic tissue surrounding the intestines, stimulating immune cells to secrete cytokines that incite inflammation throughout the body. It is recommended that animal proteins be 
severely limited or eliminated from one's diet because they are rich in arachidonic acid, which is a precursor of prostaglandins that augment different types of pain perception. For this reason, our patient was advised to supplement with flaxseed oil. Concerning the clinician's experience, acupuncture is one of the most promising tools for treating breast pain and premenstrual syndrome. When performed properly over the course of several sessions, acupuncture can eliminate nearly all pain and is one explanation for why TCM is considered superior to conventional medicine. Breast self-massage and lymphatic drainage are useful because the restriction imposed by most bras hinders proper circulation in breast tissue. This stagnation can give rise to the accumulation of toxic waste materials, so the patient was advised not to wear a bra while at home or sleeping. Choosing to wear one, however, it would be best to purchase it at the point of menstruation when breasts are at their largest. Adequate bras should accommodate breasts without subjecting them and their surrounding lymph nodes to undue pressure (evidenced by red marks on the skin). Therefore, push-up, underwire, and strapless bras must be avoided. The case reported here attests to the efficacy of TCM to treat MD over conventional options. TCM is a holistic method that slowly and consistently treats a disease and is linked to shorter recovery periods. Modern medicine, conversely, is more potent and often inflicts significant side effects. In the West, fibrocystic breast disease is typically managed with symptomatic treatments like aspirin, vitamin E, contraceptives (in the case of irregular periods), or dietary regimens that restrict caffeine or salt. For Eastern medicine, the aim is to target the root cause of a problem. Once fibrocystic breast disease is detected at even the minute's level, treatment should be carried out at a health Centre specializing in natural approaches.

\section{Conclusion}

MD patients need not endure breast pain and fibrocystic breast disease due to diagnostic and management limitations within the Western medical system. These directionless procedures result in deviating female from maintaining a healthy breast and installing negative emotions. Alternative treatments, such as TCM, address the root cause rather than the symptoms, effectively help to managing breast pain, cystic lump growth and impede cancer growth by defending against chemicals in the environment, thereby leading to a better quality of life. The author recommends for additional clinical studies comparing Western and Eastern medicine with a patient-centered approach to confirm the findings of this study.

\section{Competing Interests Statement}

The author declares that there are no competing or potential conflicts of interest.

\section{References}

American College of Clinical Thermology. ACCT approved thermography clinics [Internet]. Retrieved March 25, 2017, from http://www.thermologyonline.org. Breast_thermography_clinics.htm

Copeland, M., (1963). The treatment of mammary dysplasia with special reference to microcystic and macrocystic disease of the breast. American Journal of Surgery, 106(3), 382-395. https://doi.org/10.1016/0002-9610(63)90120-x.

Crystal, P., Strano, S. D., Shcharynski, S., \& Koretz, M. J. (2003). Using sonography to screen women with mammographically dense breasts. American Journal of Roentgenology, 181(1), 177-182. https://doi.org/10.2214/ajr.181.1.1810177.

Hoekstra, P. (2005). Quantitive digital thermology: 21st century imaging systems. Paper presented at OAND Conference; 2001; Hamilton, Ontario Institute of Medicine (US) and National Research Council (US) Committee on New Approaches to Early Detection and Diagnosis of BC, Saving Women's Lives: Strategies for Improving BC Detection and Diagnosis. Washington (DC): National Academies Press (US); 2005. 2, Benefits and Limitations of Mammography. Retrieved from https://www.ncbi.nlm.nih.gov/books/NBK22311/

Hornberger, J., Chen, S. C., Li, Q., Kakad, P., \& Quay, S. C. (2015). Proliferative epithelial disease identified in nipple aspirate fluid and risk of developing BC: A systematic review. Current Medical Research and Opinion, 31(2), 253-262. https://doi.org/10.1185/03007995.2014.988209

Kennedy, D. A., Lee, T., \& Seely, D. (2009). A comparative review of thermography as a BC screening technique. Integrative Cancer Therapies, 8(1), 9-16. https://doi.org/10.1177/1534735408326171

Snedeker, S. M., (2001). Pesticides and BC risk: A review of DDT, DDE, and dieldrin. Environmental Health Perspectives, 109(Suppl. 1), 35-47. https://doi.org/10.1289/ehp.01109s135 


\section{Copyrights}

Copyright for this article is retained by the author(s), with first publication rights granted to the journal.

This is an open-access article distributed under the terms and conditions of the Creative Commons Attribution license (http://creativecommons.org/licenses/by/4.0/). 